\title{
Análise comparativa da eficiência energética entre os ciclos de vida do gás natural comprimido e da energia termelétrica a gás para uso em automóveis
}

\author{
Mauro Pereira Hill ${ }^{1}$ e Márcio de Almeida D’Agosto ${ }^{2}$
}

\begin{abstract}
Resumo: Este artigo compara duas fontes de energia para uso veicular, considerando a cadeia de suprimentos e o uso final para cada alternativa sob o aspecto da eficiência energética e do consumo de energia. O procedimento utilizado é fundamentado na ferramenta Análise de Ciclo de Vida (ACV) e resulta numa abordagem conhecida como "well-to-wheel". Sua aplicação compara o inventário de ciclo de vida do gás natural comprimido para uso final em automóveis equipados com motor de combustão interna de ciclo Otto (ICV ${ }_{1}$ ) e o inventário de ciclo de vida da energia elétrica produzida em usinas termelétricas a gás para uso final em automóveis elétricos a bateria $\left(\mathrm{ICV}_{2}\right)$. O resultado desta análise indica que enquanto o $\mathrm{ICV}_{1}$ apresenta uma eficiência energética total de $12,4 \%$, o ICV 2 apresenta eficiência energética de 27,5\% (121\% mais eficiente do que $\left.\mathrm{ICV}_{1}\right)$. No $\mathrm{ICV}_{1}$, para promover o deslocamento de 1,5 passageiros ao longo de $1.000 \mathrm{~km}$, é necessário o consumo de 2.196,4 MJ, enquanto no $\mathrm{ICV}_{2}$ este consumo é de apenas 633,5 MJ, (o que significa que o $\mathrm{ICV}_{1}$ consome $247 \%$ mais energia do que o $\mathrm{ICV}_{2}$ para efetuar o mesmo trabalho). Estes números mostram que em termos de uso da energia, $\mathrm{ICV}_{2}$ é melhor do que o $\mathrm{ICV}_{1}$, mais usual no Brasil e no mundo, indicando a possibilidade de melhoria na aplicação do gás natural em transportes.

Palavras-chave: gás natural comprimido, análise de ciclo de vida, veículos elétricos, eficiência energética.
\end{abstract}

\begin{abstract}
This article compares two power sources for vehicular use considering the whole supply chain and final use for each alternative from the viewpoint of energy efficiency and energy consumption. The procedure by means of a well to wheel analysis, based on Life Cycle Assessment (LCA), is applied in the comparison between the life cycle inventory of the compressed natural gas used in vehicles with internal combustion engines of the Otto cycle $\left(\mathrm{LCI}_{1}\right)$ and life cycle inventory of the electricity produced from natural gas power plants to final use in the battery electric vehicles $(\mathrm{BEV})\left(\mathrm{ICV}_{2}\right)$. The analysis indicates that while $\mathrm{LCI}_{1} \mathrm{presents}$ overall efficiency of $12.4 \%$, the $\mathrm{LCI}_{2}$ indicates energy efficiency of $27.5 \%\left(121 \%\right.$ more efficient than $\left.\mathrm{LCI}_{1}\right)$. In $\mathrm{LCI}_{1}$, to promote the displacement of 1.5 passengers $1.000 \mathrm{~km}$ away, it is necessary to spend 2,196.4 MJ, while in $\mathrm{LCI}_{2}$ it must be consumed $633.5 \mathrm{MJ}$, (what means $247 \%$ better in terms of energy consumption). Those numbers show clearly that in terms of energy use, $\mathrm{LCI}_{2}$ is better than $\mathrm{LCI}_{1}$, indicating a possibility of change in order to practice a better use to natural gas application in transportation.
\end{abstract}

Keywords: compressed natural gás, life cycle assessment, electric vehicle, energy efficiency.

\section{INTRODUÇÃO}

O consumo de energia no setor de transportes no Brasil aumentou 32,3\% entre 2000 e 2009 (BEN, 2010), período no qual a participação do gás natural comprimido (GNC) no consumo de energia aumentou de $0,6 \%$ (2000) para $3,2 \%$ (2009). O uso do gás natural comprimido em automóveis é uma prática comum em países como Itália, China, EUA, Argentina, Alemanha, Irã, Índia e Brasil (NGNVCG, 2010). No Brasil, existem 1.640 .000 automóveis (NGNVCG, 2010) com motores de combustão interna (veículo a combustão interna - VCI) adaptados para GNC. No entanto, a introdução dos veículos elétricos a bateria plug in (veículo elétrico a bateria - VEB) no mercado automotivo e o fato do gás natural constituir uma fonte primária de energia que pode ser transformada em eletricidade nas usinas termelétricas, inspirou o objetivo de verificar a hipótese: "considerando o ciclo de vida das fontes de energia, o uso

\footnotetext{
${ }^{1}$ Mauro Pereira Hill, Programa de Engenharia de Transporte, Instituto Alberto Luiz Coimbra de Pós-Graduação e Pesquisa de Engenharia, Universidade Federal do Rio de Janeiro, Rio de Janeiro, RJ, Brasil. (e-mail: maurohill@ig.com.br).

${ }^{2}$ Márcio de Almeida D’Agosto, Programa de Engenharia de Transporte, Instituto Alberto Luiz Coimbra de Pós-Graduação e Pesquisa de Engenharia, Universidade Federal do Rio de Janeiro, Rio de Janeiro, RJ, Brasil. (e-mail: dagosto@pet.coppe.ufrj.br).
}

Manuscrito recebido em 25/4/2011 e aprovado para publicação em 30/5/2011. Este artigo é parte de TRANSPORTES v.19, n.2, 2011. ISSN: 2237-1346 (online). de GN em usinas termelétricas para a produção de energia elétrica para uma frota de VEBs apresenta maior eficiência energética e menor consumo de energia do que o uso de gás natural comprimido em veículos com motores de combustão interna (MCI), prática atual no Brasil e no mundo.”

Após esta introdução o trabalho apresenta sucintamente o procedimento a ser aplicado para verificação da hipótese (item 2). No item 3 apresenta-se a aplicação do procedimento e a análise dos resultados. Por fim, são apresentadas as considerações finais e sugestões.

\section{METODOLOGIA E DEFINIÇÕES}

A Figura 1 apresenta o procedimento (D'Agosto e Ribeiro, 2009) adotado para a comparação entre o inventário de ciclo de vida do gás natural comprimido para uso final em automóveis equipados com motor de combustão interna de ciclo Otto (ICV1) e o inventário de ciclo de vida da energia elétrica produzida em usinas termelétricas a gás para uso final em automóveis elétricos a bateria (ICV2), cujo entendimento será possível por meio da aplicação apresentada no item 3 deste trabalho.

Uma vez que se tenha identificado os Modelos de Ciclo de Vida (MCV), a aplicação do procedimento (Figura 1) possibilita primeiramente a determinação dos valores das eficiências energéticas (valores percentuais), para cada estágio de um $\mathrm{MCV}_{\mathrm{j}}$ associado a um $\mathrm{ICV}_{\mathrm{i}}$, gerando matrizes de eficiências energéticas que apresentam valores mínimos, médios e máximos de eficiência para cada $\mathrm{MCV}_{\mathrm{j}}$ em nível 


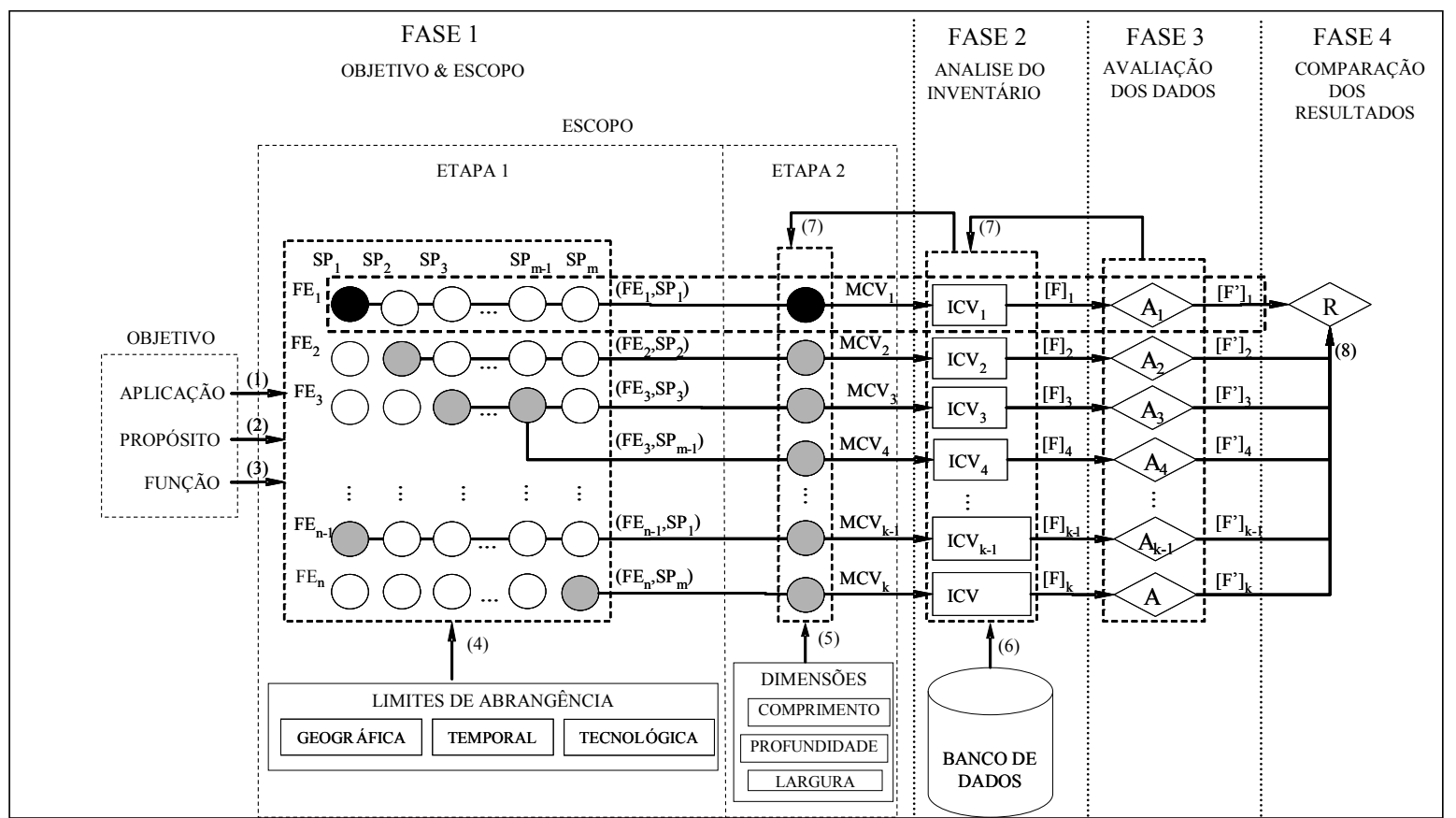

Fonte: D’Agosto e Ribeiro (2009).

Legenda: (1) Fontes de energia para transporte; (2) Comparar alternativas; (3) Produzir deslocamento; (4) Dados de restrição e relacionamento; (5) Limites do sistema de produto; (6) Dados para o inventário; (7) Dados para reavaliação; (8) Dados para comparação; FE: Fonte de energia; SP: Sistema de propulsão; MCV: Modelo de Ciclo de Vida; ICV: Inventário de Ciclo de Vida; [F]: Matriz de fluxos do ICV; A: Avaliação da qualidade dos dados; [F']: Matriz de fluxos avaliados do ICV; C: Comparação dos resultados.

Figura 1. Detalhamento das fases do procedimento do ICV para comparação de fontes de energia para transporte

de micro, meso e macro-estágios. A eficiência de um macro-estágio é obtida pela multiplicação das eficiências de seus meso-estágios, que por sua vez são obtidos a partir da multiplicação das eficiências dos micro-estágios.

O conceito de eficiência energética utilizado neste trabalho pode ser compreendido através da Figura 2, que apresenta os fluxos de energia dentro de um estágio e das equações (1) e (2). A “energia na saída" do processo é calculada através da equação (1).

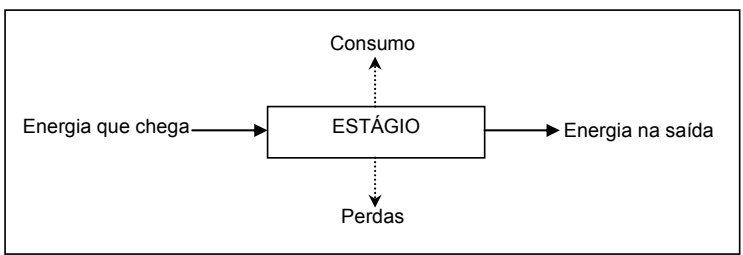

Figura 2. Fluxos de energia no estágio do ciclo de vida

$$
\begin{aligned}
& \text { Energia na saida do processo }=\text { Energia que } \\
& \text { chega ao processo }- \text { consumo }- \text { perda } \\
& \qquad\left(E_{f e}\right)=\frac{\text { Quantidade de energia na saida }}{\text { Quantidade de energia que chega }}
\end{aligned}
$$

A partir das eficiências energéticas e do rendimento energético dos veículos (uso final), é possível restituir a matriz de consumo de energia em cada estágio do ciclo de vida. Esta forma de aplicação do procedimento estabelece uma contribuição ao trabalho de D'Agosto e Ribeiro (2009) por meio de um mecanismo para o cálculo indireto dos consumos energéticos dos estágios dos ICVs na base funcional, além de enriquecer a análise no contexto do uso da energia nos ICVs envolvidos.

\section{APLICAÇÃO DO PROCEDIMENTO DE ANÁLISE DE INVENTÁRIOS DE CICLOS DE VIDA}

A seguir, apresenta-se a aplicação do procedimento tendo como base a Figura 1.

\subsection{Fase 1: Objetivo e definição do escopo}

O propósito do estudo é comparar o consumo de energia e a eficiência energética entre $\mathrm{ICV}_{1}$ e ICV $\mathrm{ICV}_{2}$, tendo como função o deslocamento de passageiros. A unidade funcional escolhida para a elaboração da matriz de consumo energético foi $\mathrm{MJ} /($ pass $\times 1.000 \mathrm{~km})$, enquanto matrizes que trazem eficiências energéticas conterão valores percentuais.

\subsubsection{Escopo - Etapa 1-Definição das alternativas de ci- clo de vida e dos limites da abordagem}

Para caracterizar as alternativas de ciclo de vida em análise foram escolhidos dois pares de fontes de energia (FE) e sistema de propulsão (SP) (Tabela 1). No caso do par (GNC,VCI), a abrangência geográfica foi definida de forma a compor a cadeia de suprimentos de GNC para seu maior produtor e consumidor nacional: o estado do Rio de Janeiro. Em 2008, o Rio de Janeiro foi responsável por 40,58\% do gás natural nacional produzido (ANP, 2010) e por $42,5 \%$ do gás consumido para fins automotivos (ABEGAS, 2009) estando as reservas gasíferas exploradas neste estado localizadas em subsolo marinho (off shore) (ANP, 2010), assim como $71 \%$ da produção nacional. A delimitação da abrangência geográfica da cadeia de suprimentos conduz à abrangência tecnológica onde serão consideradas a exploração do gás natural oriundo da bacia petrolífera de Cam- 
Tabela 1. Caracterização das alternativas

\begin{tabular}{|c|c|c|c|c|c|c|}
\hline \multicolumn{3}{|c|}{ Fonte de Energia(FE) } & \multicolumn{3}{|c|}{ Sistema de Propulsão (SP) } & \multirow{2}{*}{$\begin{array}{l}\text { Alternativas } \\
(F E, S P)\end{array}$} \\
\hline Nome & Iniciais & Caracterização & Nome & Iniciais & Caracterização & \\
\hline $\begin{array}{l}\text { Gás natural } \\
\text { comprimido }\end{array}$ & GNC & $\begin{array}{l}\text { Gás natural associado } \\
\text { ao petróleo, processa- } \\
\text { do e comprimido } \\
\text { Eletricidade } \\
\text { produzida em } \\
\text { termelétricas de } \\
\text { ciclo-combinado a } \\
\text { gás natural }\end{array}$ & $\begin{array}{l}\text { Veículo com } \\
\text { motor de } \\
\text { combustão } \\
\text { interna (1) }\end{array}$ & VCI & $\begin{array}{l}\text { Carro de passageiros } \\
\text { adaptado para admi- } \\
\text { tir GNC como prin- } \\
\text { cipal combustível } \\
\text { Carro de passageiros } \\
\text { cuja fonte de energia } \\
\text { é a eletricidade ar- } \\
\text { mazenada em bateri- } \\
\text { as }\end{array}$ & $(\mathrm{GNC}, \mathrm{VCI})$ \\
\hline
\end{tabular}

pos (norte fluminense) e o abastecimento de VCIs nos postos de combustíveis situados no município do Rio de Janeiro. O estado também possui a maior frota de veículos leves adaptados para GNC do Brasil (732.013 unidades), com $40 \%$ do total (NGNVCG, 2010). A abrangência da tecnologia do uso final (VCI) a ser considerada, está calcada no perfil da frota carioca de veículos leves existente, que considera motores alternativos de combustão interna de ignição por centelhamento e quatro tempos - ciclo Otto (D'Agosto e Ribeiro, 2007, 2009). Foram considerados também aspectos técnicos (autonomia, velocidade máxima, potência do motor, peso, entre outros) que se aproximem da tecnologia escolhida para o VEB a fim de se comparar usos finais com perfil semelhante.

Para o par (EE,VEB), considera-se que sua abrangência geográfica inicia-se também na exploração e produção de gás natural na Bacia de Campos. As etapas de exploração, produção, transferência, processamento, transporte e distribuição de GN são as mesmas do ciclo (GNC, VCI), com a diferença que o gás processado passa a ser destinado para termelétricas a gás localizadas no estado do Rio de Janeiro. Em 2008, 66\% do gás natural consumido no estado do Rio de Janeiro destinou-se a geração e cogeração de energia elétrica do país (ABEGAS, 2009). As quatro termelétricas mais potentes do país utilizam o gás natural como combustível e estão localizadas no Rio de Janeiro, constituindo assim o maior parque de geração de energia elétrica a partir de termelétricas a GN do país. A energia elétrica produzida é transportada em alta tensão e distribuída para a rede elétrica do município do Rio de Janeiro. A temporalidade associada ao uso final é o momento presente, pois a tecnologia do VEB passa por um momento histórico que favorece seu desenvolvimento, fazendo com que qualquer passado recente possa representar tecnologias obsoletas. A tecnologia veicular associada ao uso final da energia elétrica deve ser passível de comparações com o veículo equipado com motor de combustão interna escolhido para a alternativa de ciclo de vida (GNC, VCI).

O perfil do automóvel a ser considerado foi definido a partir das especificações técnicas de oito modelos de veículos populares utilizados no Rio de Janeiro. As médias de potência, peso, autonomia, velocidade máxima podem ser encontradas da Tabela 1. Determinou-se o rendimento de $12 \mathrm{~km} / \mathrm{m}^{3}$ em tráfego urbano para o VCI por meio de pesquisa feita no Rio de Janeiro (Hill, 2010).

Seguindo o escopo do trabalho, alguns modelos de veículos elétricos foram pesquisados, sendo que o automóvel denominado iMiev, produzido pela Mitsubishi, foi escolhido por ser o veículo cujos parâmetros técnicos e construti- vos possuem maior semelhança com os valores definidos para o VCI (Tabela 1). Outro motivo que influenciou a escolha do iMiev deve-se ao uso da bateria de células de íons-lítio que possui maior densidade energética além de ser a mais difundida atualmente pelos fabricantes de VEB (Hill, 2010). Numa tomada de $220 \mathrm{~V}$ o tempo de recarga das baterias é de 7 horas aproximadamente. Suas baterias permitem cerca de 1.000 recargas (MITSUBISHI, 2009). O consumo energético do iMiev considerado foi de $0,125 \mathrm{KWh} / \mathrm{km}$ (MITSUBISHI, 2010). A autonomia do iMiev é de $160 \mathrm{~km}$ por carga completa (Tabela 1), valor superior ao do veículo com motor de combustão interna adaptado para GNC, que com cilindro de $10 \mathrm{~m}^{3}$ possui autonomia entre 115 e $158 \mathrm{~km}$. Na área urbana é comum que se trafegue entre 20 a $40 \mathrm{~km}$ por dia, o que indica que a autonomia de $160 \mathrm{~km}$ do VEB pode ser, num primeiro momento, satisfatória.

\subsubsection{Escopo - Etapa 2 - Descrição dos modelos de ciclo de vida}

Esta etapa apresenta e descreve os modelos de ciclo de vida (MCV) associado aos pares (GNC, VCI) e (EE, VEB). Os dois modelos de ciclos de vida apresentados neste item se "estendem" desde o meso-estágio da produção de matériaprima até ao de uso final. A "largura" foi dimensionada de acordo com o nível 1, que considera a eficiência energética diretamente associada aos micro-estágios do ciclo de vida. Para efeito de análises comparativas entre ciclos de vida, a "profundidade" escolhida foi a de meso-estágios, pois permite melhor eqüidade de comparação entre alternativas. As Figuras 3 e 4 apresentam o MCV associado à alternativa (GNC, VCI) e (EE,VEB), respectivamente.

\subsection{Fase 2: Inventários de ciclos de vida}

Para a elaboração das matrizes que trazem o inventário da eficiência energética dos ciclos de vida envolvidos, mostrados nas Tabelas 2 e 3, dados foram colhidos através de entrevistas pessoais com profissionais do respectivo setor envolvido, artigos técnicos, documentos e sites oficiais.

\subsection{Fase 3. Avaliação dos dados}

A avaliação dos dados sobre eficiência energética (Tabelas 2 e 3) foi feita através do seu confronto com valores obtidos por meio de revisão bibliográfica, de forma a verificar a sua coerência e consistência. Ainda que os trabalhos mencionados tenham sido feitos em áreas geográficas e momentos distintos, a semelhança entre os valores reflete de forma positiva o resultado aqui obtido, indicando coerência e consistência. 


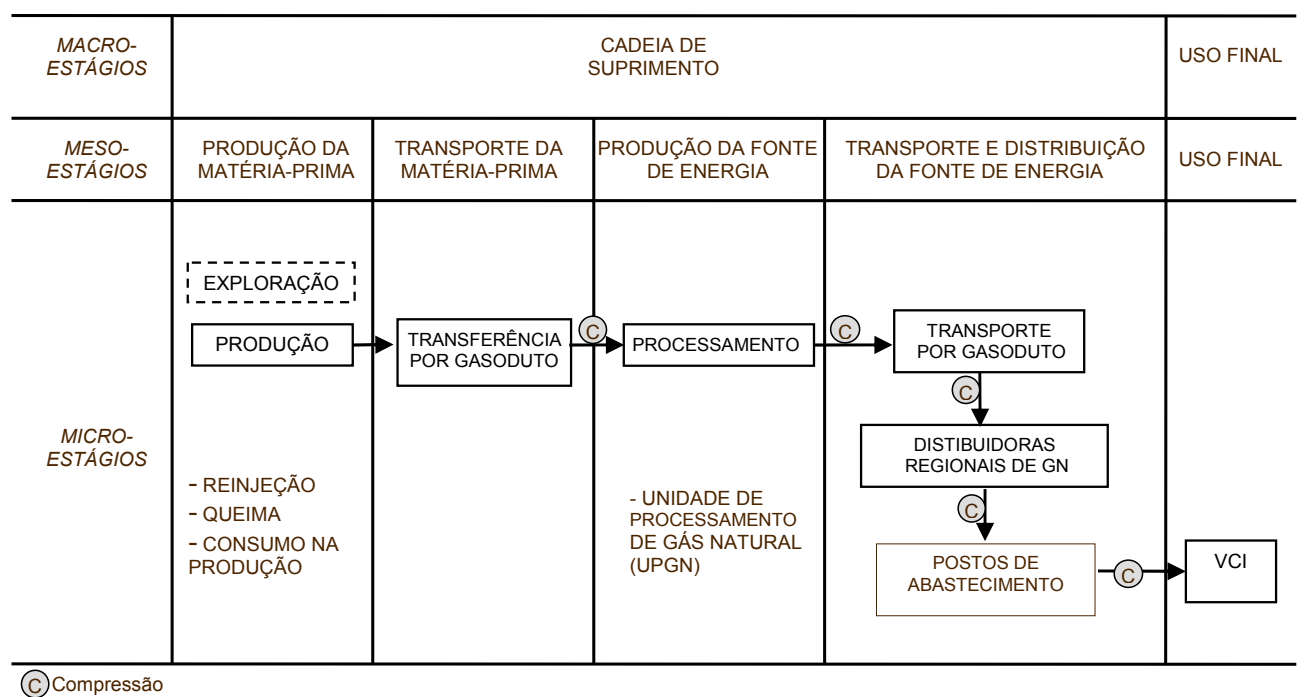

Figura 3. Modelo de Ciclo de Vida (MCV) associado à alternativa (GNC, VCI)

\begin{tabular}{|c|c|c|c|c|c|}
\hline $\begin{array}{l}\text { MACRO- } \\
\text { ESTÁGIOS }\end{array}$ & \multicolumn{4}{|c|}{$\begin{array}{l}\text { CADEIA DE } \\
\text { SUPRIMENTO }\end{array}$} & USO FINAL \\
\hline $\begin{array}{l}\text { MESO- } \\
\text { ESTÁGIOS }\end{array}$ & $\begin{array}{l}\text { PRODUÇÃO DA } \\
\text { MATERRIA-PRIMA }\end{array}$ & $\begin{array}{l}\text { TRANSPORTE DA } \\
\text { MATERRIA-PRIMA }\end{array}$ & $\begin{array}{c}\text { PRODUÇÃO DA FONTE DE } \\
\text { ENERGIA }\end{array}$ & $\begin{array}{l}\text { TRANSPORTE E DISTRIBUIÇÃO DA } \\
\text { FONTE DE ENERGIA }\end{array}$ & USO FINAL \\
\hline $\begin{array}{c}\text { MICRO- } \\
\text { ESTÁGIOS }\end{array}$ & $\begin{array}{l}\text { PRANSFERÉNCIA } \\
\text { POR GASODUTOS } \\
\text { PROCESSAMENTO } \\
\text { - REINJEÇÃO } \\
\text { - QUEIMA } \\
\text { - CONSUMO NA } \\
\text { PRODUÇĀO } \\
\text { - PROCESSAMENTO } \\
\text { PRIMARIO }\end{array}$ & $\begin{array}{c}\text { TRANSPORTE } \\
\text { POR GASODUTOS } \\
\text { DISTRIBUIDORAS } \\
\text { REGIONAIS DE GN }\end{array}$ & USINAS & $\begin{array}{c}\text { REDE DE } \\
\text { DISTRIBUIÇĀO DE } \\
\text { ELETRICIDADE }\end{array}$ & VEB \\
\hline
\end{tabular}

Figura 4. Modelo de Ciclo de Vida (MCV) do par (EE, VEB)

Tabela 2. Inventário de energia do ciclo de vida para alternativa (CNG, VCI)

\begin{tabular}{|c|c|c|c|c|c|c|c|c|}
\hline & \multirow[b]{2}{*}{ Macro-estágios } & \multirow[b]{2}{*}{ Meso-estágios } & \multirow[b]{2}{*}{ Micro-estágios } & \multicolumn{3}{|c|}{ Eficiência energética\% } & \multirow{2}{*}{$\begin{array}{l}\text { Consumo } \\
M J /(\text { pass } \times \\
1000 \mathrm{~km}) \\
\end{array}$} & \multirow{2}{*}{$\begin{array}{l}\text { Participação } \\
\text { \% ICV } \\
\text { consumo } \\
\end{array}$} \\
\hline & & & & Min & Med & $\operatorname{Max}$ & & \\
\hline \multirow{13}{*}{ 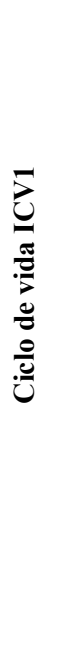 } & \multirow{11}{*}{ 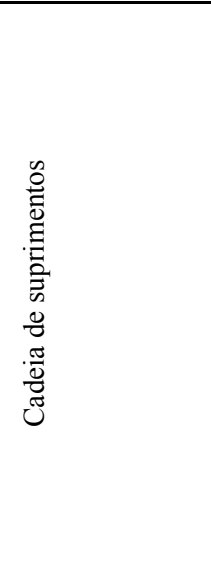 } & Produção de & Exploração de GN & $99,6 \%$ & $99,7 \%$ & $99,8 \%$ & 7,5 & $0,3 \%$ \\
\hline & & matéria prima & Produção de GN & $92,9 \%$ & $95,7 \%$ & $97,1 \%$ & 107,5 & $4,9 \%$ \\
\hline & & Total & & $92,5 \%$ & $95,7 \%$ & $96,9 \%$ & 115 & $5,2 \%$ \\
\hline & & $\begin{array}{l}\text { Transporte de } \\
\text { matéria prima }\end{array}$ & $\begin{array}{l}\text { Transferência de } \\
\text { GN }\end{array}$ & $95,0 \%$ & $96,5 \%$ & $98,0 \%$ & 83,7 & $3,8 \%$ \\
\hline & & Total & & $95,0 \%$ & $96,5 \%$ & $98,0 \%$ & 83,7 & $3,8 \%$ \\
\hline & & $\begin{array}{l}\text { Produção da } \\
\text { fonte de } \\
\text { energia }\end{array}$ & Tratamento de GN & $97,6 \%$ & $97,6 \%$ & $97,6 \%$ & 55,4 & $2,5 \%$ \\
\hline & & Total & & $97,6 \%$ & $97,6 \%$ & $97,6 \%$ & 55,4 & $2,5 \%$ \\
\hline & & & Transporte de GN & $92,0 \%$ & $93,5 \%$ & $95,0 \%$ & 146,5 & $6,7 \%$ \\
\hline & & $\begin{array}{l}\text { Transporte e } \\
\text { distribuição da } \\
\text { fonte de ener- }\end{array}$ & $\begin{array}{l}\text { Distribuição de } \\
\text { GN }\end{array}$ & $100,0 \%$ & $100,0 \%$ & $100,0 \%$ & 0 & $0,0 \%$ \\
\hline & & gia & GNC & $98,1 \%$ & $98,4 \%$ & $98,6 \%$ & 33,7 & $1,5 \%$ \\
\hline & & Total & & $90,3 \%$ & $92,0 \%$ & $93,7 \%$ & 180,2 & $8,2 \%$ \\
\hline & Total & & & $77,4 \%$ & $82,9 \%$ & $86,8 \%$ & 434,3 & $19,8 \%$ \\
\hline & Uso final & Uso final & VCI & $15,0 \%$ & $15,0 \%$ & $15,0 \%$ & 1762,1 & $80,2 \%$ \\
\hline Total & & & & $11,6 \%$ & $12,4 \%$ & $13,0 \%$ & 2196,4 & $100,0 \%$ \\
\hline
\end{tabular}


Tabela 3. Inventário de energia do ciclo de vida para alternativa (EE, VEB)

\begin{tabular}{|c|c|c|c|c|c|c|c|c|}
\hline & \multirow[b]{2}{*}{ Macro-estágio } & \multirow[b]{2}{*}{ Meso-estágios } & \multirow[b]{2}{*}{ Micro-estágios } & \multicolumn{3}{|c|}{ Eficiência energética\% } & \multirow{2}{*}{$\begin{array}{l}\text { Consumo } \\
\text { MJ/(pass } \times \\
1000 \mathrm{~km})\end{array}$} & \multirow{2}{*}{$\begin{array}{l}\text { Participação } \\
\text { \% ICV } \\
\text { consumo }\end{array}$} \\
\hline & & & & Min & Med & $\operatorname{Max}$ & & \\
\hline \multirow{16}{*}{ 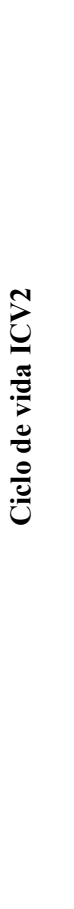 } & \multirow{14}{*}{ 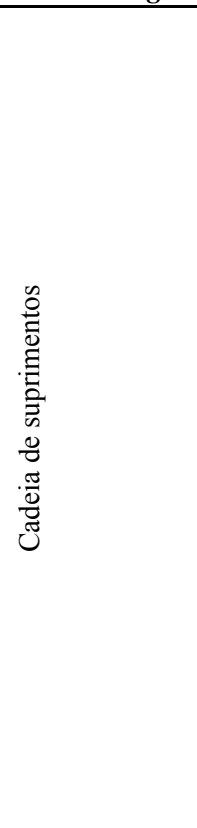 } & \multirow{5}{*}{$\begin{array}{l}\text { Produção de } \\
\text { matéria prima } \\
\text { Total }\end{array}$} & Exploração de GN & $99,6 \%$ & $99,7 \%$ & $99,8 \%$ & 2,6 & $0,4 \%$ \\
\hline & & & Produção de GN & $92,9 \%$ & $95,7 \%$ & $97,1 \%$ & 37,4 & $5,9 \%$ \\
\hline & & & $\begin{array}{l}\text { Transferência de } \\
\text { GN }\end{array}$ & $95,0 \%$ & $96,5 \%$ & $98,0 \%$ & 29,2 & $4,6 \%$ \\
\hline & & & $\begin{array}{l}\text { Processamento de } \\
\text { GN }\end{array}$ & $97,6 \%$ & $97,6 \%$ & $97,6 \%$ & 19,3 & $3,1 \%$ \\
\hline & & & & $85,8 \%$ & $89,9 \%$ & $92,7 \%$ & 88,5 & $14,0 \%$ \\
\hline & & \multirow{3}{*}{$\begin{array}{l}\text { Transporte de } \\
\text { matéria prima } \\
\text { Total }\end{array}$} & $\begin{array}{l}\text { Transporte de GN } \\
\text { processado } \\
\text { Distribuição de }\end{array}$ & $94,0 \%$ & $95,5 \%$ & $97,0 \%$ & 35,3 & $5,6 \%$ \\
\hline & & & GN processado & $100,0 \%$ & $100,0 \%$ & $100,0 \%$ & 0 & $0,0 \%$ \\
\hline & & & & $94,0 \%$ & $95,5 \%$ & $97,0 \%$ & 35,3 & $5,6 \%$ \\
\hline & & \multirow{2}{*}{$\begin{array}{l}\text { Produção da } \\
\text { fonte de ener- } \\
\text { gia } \\
\text { Total }\end{array}$} & $\begin{array}{l}\text { Produção de } \\
\text { energia elétrica }\end{array}$ & $48,0 \%$ & $51,5 \%$ & $56,0 \%$ & 363,6 & $57,4 \%$ \\
\hline & & & & $48,0 \%$ & $51,5 \%$ & $56,0 \%$ & 363,6 & $57,4 \%$ \\
\hline & & \multirow{4}{*}{$\begin{array}{l}\text { Transporte e } \\
\text { distribuição da } \\
\text { fonte de ener- } \\
\text { gia } \\
\text { Total }\end{array}$} & $\begin{array}{l}\text { Transmissão de } \\
\text { EE }\end{array}$ & $93,0 \%$ & $96,5 \%$ & $98,0 \%$ & 13,5 & $2,1 \%$ \\
\hline & & & $\begin{array}{l}\text { Distribuição de } \\
\text { EE } \\
\text { Carregamento de }\end{array}$ & $92,0 \%$ & $93,1 \%$ & $94,2 \%$ & 25,7 & $4,1 \%$ \\
\hline & & & EE & $83,0 \%$ & $86,5 \%$ & $90,0 \%$ & 46,8 & $7,4 \%$ \\
\hline & & & & $71,0 \%$ & $77,7 \%$ & $83,1 \%$ & 86,0 & $13,6 \%$ \\
\hline & Total & & & $27,5 \%$ & $34,3 \%$ & $41,8 \%$ & 573,5 & $90,5 \%$ \\
\hline & Uso final & Uso final & VEB & $80,0 \%$ & $80,0 \%$ & $80,0 \%$ & 60 & $9,5 \%$ \\
\hline Total & & & & $22,0 \%$ & $27,5 \%$ & $33,5 \%$ & 633,5 & $100,0 \%$ \\
\hline
\end{tabular}

Hekkert et al. (2005), identificaram valores de 96,9\% de eficiência energética na exploração do GNC, além de $92,8 \%$ para a produção e $96,6 \%$ no transporte de GNC. Estes valores indicaram uma eficiência de $87 \%$ na cadeia de suprimentos do GNC. Este mesmo estudo aponta que eficiência energética do VEB está entre $75 \%$ e $84 \%$. A Agência Internacional de Energia (IEA, 2008) divulgou valores máximos $(94 \%)$ e mínimos $(86,5 \%)$ para a eficiência energética da cadeia de suprimentos do GNC. D'Agosto e Ribeiro (2007, 2009) apresentam as eficiências energéticas da cadeia de suprimentos do GNC, que está entre $86,5 \%$ e $94 \%$. Segundo Handa e Yoshida (2007), a eficiência energética final obtida para o ciclo de vida do GNC para geração de eletricidade e uso final em veículos elétricos a bateria é $29 \%$, próximo dos $27,5 \%$ apresentados neste trabalho. Ramos-Real et al. (2007) informam que a eficiência da termelétrica de ciclo combinado pode variar de acordo com a taxa de operação, quanto mais horas se opera, maior é a eficiência obtida. Esta variação ficou entre $45 \%$ e $60 \%$. A Tabela 3 mostra que o meso-estágio referente à produção de energia elétrica foi o que mostrou menor valor de eficiência energética no $\mathrm{ICV}_{2}$, com valores entre $48 \%$ e 56 .

\subsection{Fase 4 - Comparação dos resultados}

Este item trata da comparação dos resultados obtidos, considerando-se a eficiência energética e o consumo de energia.

\subsubsection{Comparação dos resultados sobre eficiências ener- géticas do $I C V_{1}$ e ICV}

Tomando-se como base comparativa os valores médios totais das eficiências energéticas dos $\operatorname{ICV}_{1}(12,4 \%)$ e $\mathrm{ICV}_{2}$ $(27,5 \%)$, pode-se afirmar que o $\mathrm{ICV}_{2}$ é $121 \%$ mais eficiente do que o $\mathrm{ICV}_{1}$ (Figura 5). Em relação às eficiências energé- ticas dos ICV's apresentados, esse resultado confirma parcialmente a hipótese (a mesma será integralmente confirmada no item 3.4.2 deste trabalho) apresentada na introdução deste artigo, onde "considerando o ciclo de vida das fontes de energia, o uso de GN em usinas termelétricas para a produção de energia elétrica para uma frota VEB apresenta maior eficiência energética" ... "do que o uso de gás natural comprimido em motores de combustão interna (MCI), prática atual no Brasil”.

O baixo valor de eficiência do VCI (15\%) compromete a alta eficiência da cadeia de suprimentos $(82,7 \%)$ resultando em $12,4 \%$ de eficiência para o $\mathrm{ICV}_{1}$. Para o $\mathrm{ICV}_{2}$ as eficiências apresentadas em seus meso-estágios (Tabela 3) informam o valor de $34,3 \%$ para a cadeia de suprimentos da energia termelétrica e $80 \%$ de eficiência do VEB conduzindo a $27,5 \%$ de eficiência no $\mathrm{ICV}_{2}$.

No $\mathrm{ICV}_{1}$ o meso-estágio crítico quanto à eficiência energética é o uso final, pois todos os meso-estágios que o antecedem se mostram com eficiências superiores aos análogos do $\mathrm{ICV}_{2}$ (Figura 6). Numa análise entre meso-estágios (Figura 6), verifica-se que o $\mathrm{ICV}_{1}$ leva vantagem, sob a ótica da eficiência energética, em todos os meso-estágios da cadeia de suprimentos. Isso ocorre desde a "produção da matéria-prima" até o "transporte e distribuição" da fonte de energia. Destaca-se o meso-estágio de "produção da fonte de energia", onde a alta eficiência na produção de gás natural nas UPGNs $(97,6 \%)$ no $\mathrm{ICV}_{1}$ se destaca diante da eficiência de 51,5\% alcançada pelas termelétricas $\mathrm{ICV}_{2}$. Uma diferença considerável também ocorre no meso-estágio de "transporte e a distribuição da fonte de energia" refletindo maior eficiência no $\operatorname{ICV}_{1}(92 \%)$, contra o $\operatorname{ICV}_{2}(77 \%)$. 


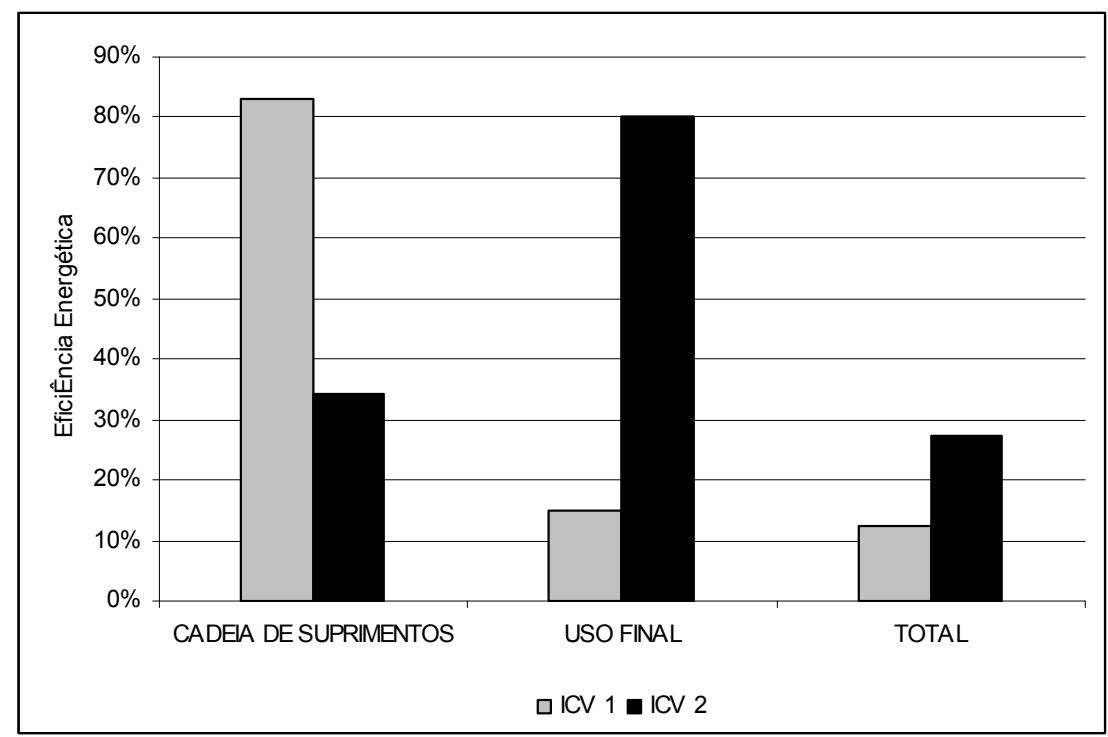

Figura 5. Comparação das eficiências energéticas dos ICV1 e ICV2 em macro-estágios

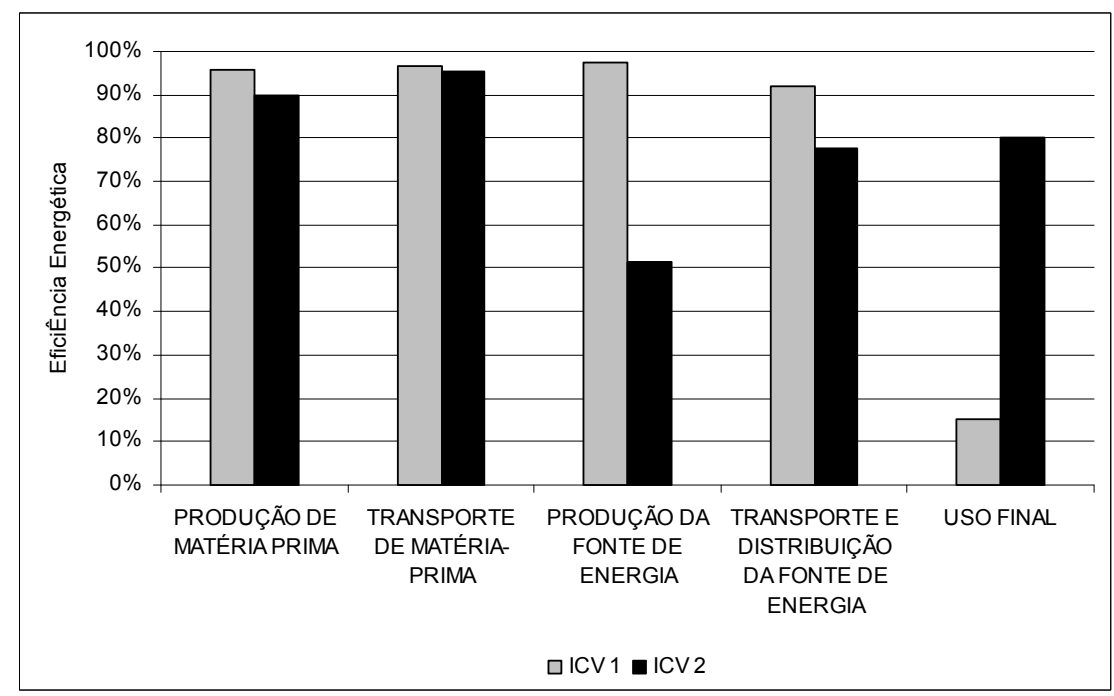

Figura 6. Comparação das eficiências energéticas dos ICV1 e ICV2 - meso-estágios

\subsubsection{Comparação dos resultados do consumo de energia no $I C V_{1}$ e $I C V_{2}$}

Os valores de consumo energético de cada inventário, em $\mathrm{MJ} /($ pass $\times 1.000 \mathrm{~km})$, contidos nas Tabelas 2 e 3 , mostram resultados sob a ótica do real aproveitamento da energia nos ciclos de vidas analisados, que até então estavam representados em valores percentuais de eficiências energéticas.

Para calcular os valores de consumo de energia no $\mathrm{ICV}_{1}$, foi considerado que para um VCI (com rendimento médio de $12 \mathrm{~km} / \mathrm{m}^{3}$, transportando 1,5 passageiro) se deslocar $1.000 \mathrm{~km}$, necessita ser abastecido com 2.073 MJ de GNC, sendo que apenas $15 \%$ disso é, de fato, transformado em trabalho. Para prover esse deslocamento, o gás natural deve passar por todas as etapas da cadeia de suprimentos do GNC e pela tecnologia que envolve o VCI. O consumo do VCI e das eficiências energéticas médias obtidas para o $\mathrm{ICV}_{1}$ permitiram a elaboração tanto do consumo energético de cada estágio do $\mathrm{ICV}_{1}$ quanto da atribuição da participação de cada um dos estágios no consumo total de energia no ciclo de vida (Tabela 2). Como resultado imediato, foi possível verificar que o estágio de uso final se destaca como maior consumidor de energia com 1.762 $\mathrm{MJ} /($ pass $\times 1000 \mathrm{~km})$, o que representa $80 \%$ do total de energia demandada pelo $\mathrm{ICV}_{1}$.

O consumo energético do iMiev está em torno de 0,125 $\mathrm{kWh} / \mathrm{km}$, ou $0,45 \mathrm{MJ} / \mathrm{km}$. Transportando 1,5 passageiro ao longo de $1.000 \mathrm{~km}$, necessita ser abastecido com $300 \mathrm{MJ}$ de energia elétrica, sendo que $80 \%$ disso é de fato transformado em trabalho. O consumo do VEB e das eficiências energéticas médias mostradas na Tabela 3 possibilitou determinar o consumo de energia dos estágios do $\mathrm{ICV}_{2}$ e a respectiva parcela que cada um deles detém no consumo total de energia no ciclo de vida. Acerca dos valores de consumos de energia apresentados pelo $\mathrm{ICV}_{2}$, o meso-estágio de transporte de matéria-prima (MP) consome apenas 5,6\% do total consumido pelo ciclo de vida, enquanto a produção de eletricidade por termelétricas é o principal estágio consumidor de energia em relação aos demais, com 57,4\%. 


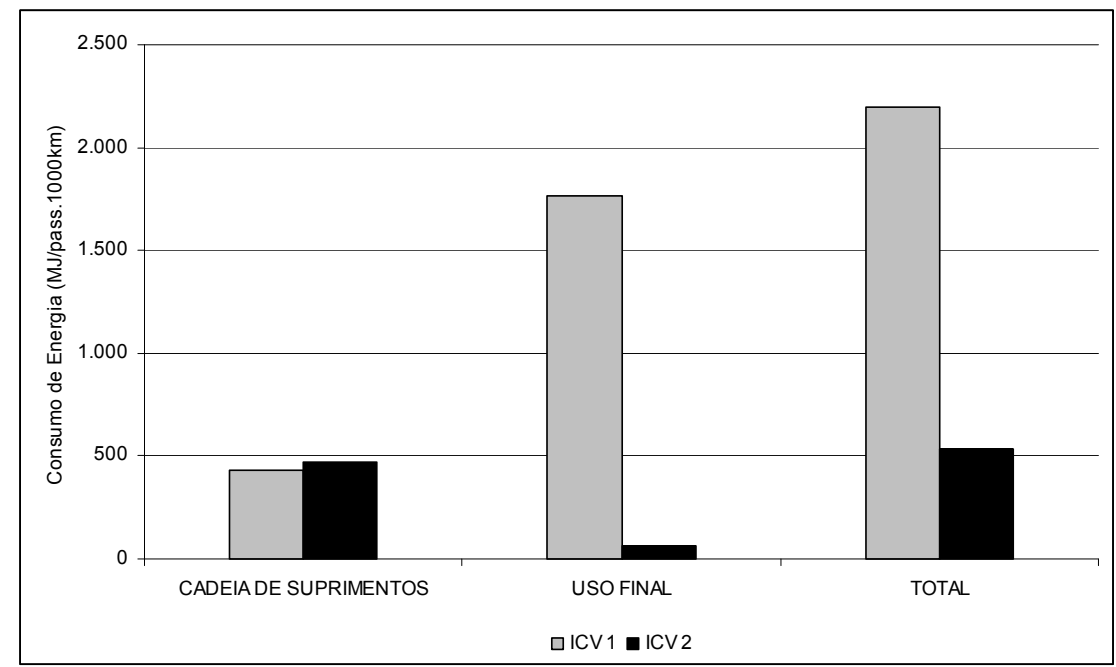

Figura 7. Consumo energético dos ICV1 e ICV2 - na profundidade de macro-estágios

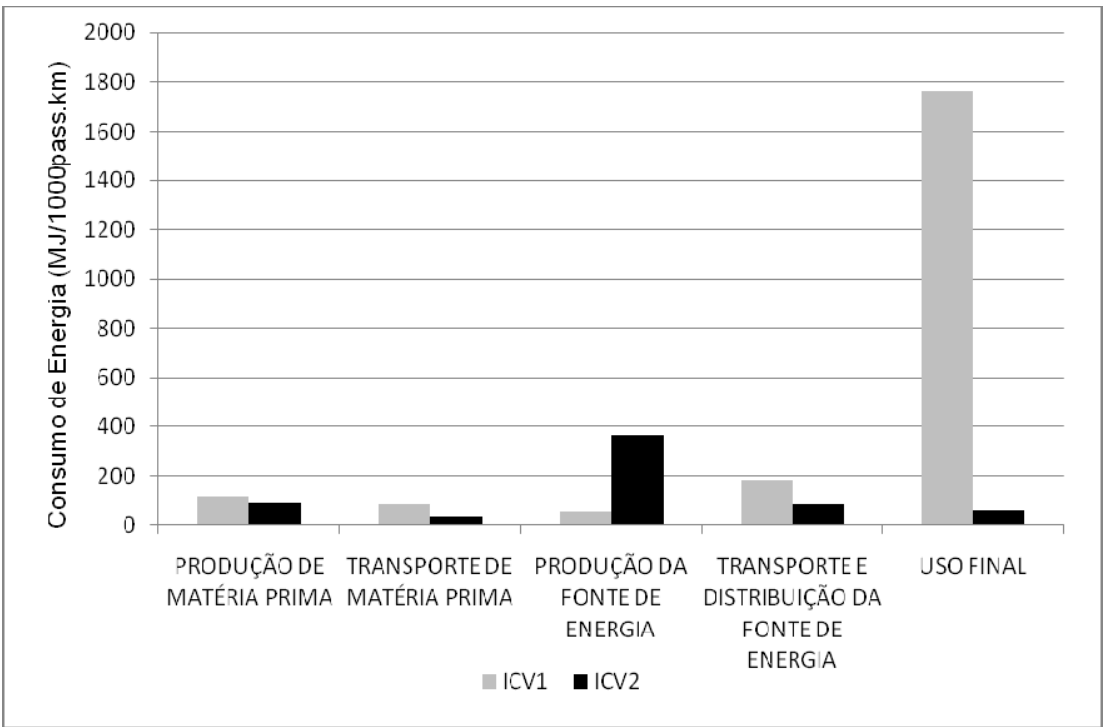

Figura 8. Comparação do consumo energético dos ICV1 e ICV2 - meso-estágios

$\mathrm{O}$ consumo de energia total do $\mathrm{ICV}_{1}$ foi de $2.196,4$ $\mathrm{MJ} /($ pass $\times 1000 \mathrm{~km})$, enquanto no $\mathrm{ICV}_{2}$ este valor foi de $633,5 \mathrm{MJ} /($ pass $\times 1000 \mathrm{~km})$; uma diferença de $247 \%$. Esse resultado completa a confirmação da hipótese inicial (apresentada na introdução deste artigo) no que tange ao menor consumo de energia do $\mathrm{ICV}_{2}$ se comparado ao $\mathrm{ICV}_{1}$ uma vez que "considerando o ciclo de vida das fontes de energia, o uso de GN em usinas termelétricas para a produção de energia elétrica para uma frota de automóveis elétricos a bateria apresenta" ... "menor consumo de energia do que a utilização em prática atualmente, que é a do uso de gás natural comprimido em motores de combustão interna (MCI)".

Observando o consumo energético do $\mathrm{ICV}_{2}$, Figura 7, em nível de macro-estágios é possível verificar que $90,5 \%$ da energia é consumida pela cadeia de suprimentos e $9,5 \%$ pelo uso final. Esta situação se inverte quando se observa o consumo energético em $\mathrm{ICV}_{1}$ onde a cadeia de suprimentos representa $20 \%$ da energia total e o uso final $80 \%$.

A partir da Figura 8 pode-se verificar que o consumo de energia pelo estágio do uso final no $\mathrm{ICV}_{1}$ é o maior entre seus meso-estágios, enquanto no $\mathrm{ICV}_{2}$, este valor é atribuído à produção da fonte de energia.
Embora a cadeia de suprimentos do $\mathrm{ICV}_{1}$ seja mais eficiente em relação à do $\mathrm{ICV}_{2}$, a alta demanda de energia do VCI acarreta altos valores absolutos de consumo em todos os meso-estágios anteriores ao uso final. Ou seja, numa primeira análise, uma cadeia de suprimentos seja relativamente mais eficiente do que outra, o alto consumo no estágio de uso final pode comprometer essa vantagem, afetando o consumo de energia em todos os estágios anteriores podendo resultar num alto consumo de energia global do ICV.

Observa-se a vantagem energética do VEB associada não apenas em relação à eficiência energética $(15 \%$ do VCI, contra $80 \%$ do VEB), mas principalmente em relação à quantidade de energia requerida por quilômetro percorrido, indicando uma grande vantagem do VEB sob este aspecto. No VCI, para promover o deslocamento de 1,5 passageiros em $1.000 \mathrm{~km}$ de distância, é necessário abastecê-lo com 2.073MJ de gás natural. Para o VEB percorrer esta mesma distância, com os mesmos 1,5 passageiros, ele deve ser abastecido com apenas $300 \mathrm{MJ}$ de eletricidade.

\section{CONSIDERAÇÕES FINAIS E SUGESTÕES}

$\mathrm{O}$ resultado deste estudo comparativo favorece a implemen- 
tação do modelo de ciclo de vida referente à alternativa (EE,VEB) como alternativa para melhoria em $247 \%$ do aproveitamento energético do gás natural da Bacia de Campos como fonte de energia para automóveis em tráfego urbano na cidade do Rio de Janeiro. O modelo de ciclo de vida que se destacou, implica no direcionamento do GN para a produção de energia elétrica nas termelétricas de ciclo combinado e na substituição gradativa da frota de veículos do Rio de Janeiro, que hoje são VCIs, para VEBs.

Conforme apresentado nos itens 3.4.1 e 3.4.2., pode-se considerar que principal objetivo do trabalho foi alcançado por meio da verificação da hipótese estabelecida na introdução.

Além da vantagem do melhor aproveitamento da energia, para o consumidor final, o consumo energético do iMiev significa um custo de $\mathrm{R} \$ 0,03892 / \mathrm{km}$ para a realidade de um abastecimento numa residência no Rio de Janeiro em dezembro de 2009, enquanto o custo do de se abastecer um VCI para a mesma realidade é em torno de R\$ $0,12 / \mathrm{km}$ (Hill, 2010). Esta análise de custo considera o preço final da energia elétrica atribuído à matriz brasileira de geração de eletricidade, onde cerca de $72 \%$ é proveniente de hidrelétricas. Porém, matrizes baseadas em termelétricas podem implicar em maiores preços ao consumidor de energia elétrica.

Outras vantagens, não verificadas neste trabalho, podem ser obtidas com a introdução da frota de veículos elétricos: menor poluição atmosférica global pela redução das emissões de $\mathrm{CO}_{2}$; menor poluição atmosférica local por redução das emissões de monóxido de carbono (CO), material particulado (MP) e hidrocarbonetos (HC); além da redução da poluição sonora provocada pelos motores de combustão interna (MITSUBISHI, 2009).

Para um trabalho mais completo sobre a comparação dos ciclos de vida aqui apresentados, recomenda-se incluir além da análise energética, informações sobre emissão de poluentes atmosféricos, emissão de resíduos sólidos e líquidos e consumo de água.

Adicionalmente, entende-se que para uma recomendação mais consistente sobre o uso de veículos elétricos a bateria (VEB), os valores de aquisição e os custos de operação destes veículos devem ser considerados. Impulsionados pelos argumentos de preservação ambiental e desenvolvimento sustentável, países como EUA, Holanda e Portugal já oferecem incentivos (descontos na aquisição, isenção de impostos, tarifas e pedágios) para estimular o uso de veículos elétricos (Hill, 2010). Estes incentivos são fundamentais para impulsionar o mercado dos VEBs, combinando a participação e interesse de diversos setores como: ambiental, de energia, automobilístico e governo.

Conforme ocorra a gradativa conquista de mercado por parte dos VEBs, a infra-estrutura voltada para seu abastecimento deve ser ampliada. As distribuidoras de energia deverão sofrer adequações para suportarem mudanças no comportamento de consumo de energia elétrica, assim como também deverão sofrer adequações os estacionamentos em geral (garagens de prédios residenciais e comerciais, de shoppings e supermercados etc.) e postos de combustíveis.

\section{REFERÊNCIAS BIBLIOGRÁFICAS}

BEN (2010) Balanço Energético Nacional 2010, Ministério de Minas e Energia, Brasília, DF. Disponível em: <www.mme.gov.br> (Acessado em 12/06/2010).
D’Agosto, M. A. e S. K. Ribeiro (2007) Procedimento de análise de ciclo de vida para comparação de fontes de energia para o transporte no Brasil. Anais do XXI Congresso de Ensino e Pesquisa em Transportes, ANPET, Rio de Janeiro, v. 1, p. 1-12.

D'Agosto, M. A. e S. K. Ribeiro (2009) Assessing total and renewable energy in Brazilian automotive fuels. A life cycle inventory (LCI) approach. Renewable and Sustainable Energy Reviews, v. 13, n. 10 , p. $1326-1337$.

NGNVCG (2010) Folha do GNV, NGV Communications Group, ano X, n. 110. Rio de Janeiro, Brasil. Disponível em: $<$ www.ngvgroup.com $>$ (Acessado em 02/07/2010).

ANP (2010) Anuário Estatístico Brasileiro do Petróleo, Gás e Biocombustíveis 2009. Agência Nacional do Petróleo, Gás e Biocombustíveis. Rio de Janeiro, RJ.

MITSUBISHI (2009) Informações sobre o veículo iMiEV, Mitsubishi Motors. Disponível em: <www.mitsubishi-motors.com/special/ev/> (Acessado em 10/12/2009).

MITSUBISHI (2010) Ficha técnica do veículo MiEV, Mitsubishi Motors. Disponível em: <www.mitsubishi-motors.com/special/ev/whatis/ index.html $>$ (Acessado em 02/02/2010).

IEA (2008) Energy Efficiency Indicators for Public Electricity Production from Fossil Fuels 2008. International Energy Agency, Paris, França.

Hekkert M. P., F. H. J. F. Hendriks, A. P.C. Faaij e M. L. Neelis (2005) Natural gas as an alternative to crude oil in automotive fuel chains well-to-wheel analysis and transition strategy development. Energy Policy, v. 33, n. 5, p 579-594.

Ramos-Real F. J.; J. C. Moreno-Piquero e J. M. Ramos-Henríquez (2007) The effects of introducing natural gás in the Canary Islands for electricity generation. Energy Policy, v. 35, n. 7, p. 3925-3935.

Handa, K. e Yoshida, H. (2007) Development of Nest-Generation Electric Vehicle "iMiev", Mitsubishi Motors-Technical Review, n 19.

Hill, M. P. (2010) Uso de energia em transporte: análise comparativa da eficiência energética entre os ciclos de vida do gás natural veicular comprimido e da energia termelétrica a gás para uso final em automóveis leves. Dissertação (mestrado), COPPE/UFRJ, Rio de Janeiro, RJ. 